\title{
Calibration of primary measuring converter by using of modeling and experimental evaluation of the initial data
}

\author{
Vladimir Skliarov ${ }^{1,2^{*}}$, Konstantin Muentean $^{1}$, and Eugen Timofeev ${ }^{1}$ \\ ${ }^{1}$ National Scientific Centre „Institute of Metrology“, 42, Myronosytska str., 61002, Kharkiv, Ukraine \\ ${ }^{2}$ O.M. Beketov National University of Urban Economy in Kharkiv, 17, Marshal Bazhanov str., 610002, Kharkiv, Ukraine
}

\begin{abstract}
The main target of this work is to decrease the uncertainty of measurement of laser beam power by isoperibolic calorimeters. Measurement and calibration operating regimes of the isoperibolic calorimeter are investigated experimentally. Temperature transients during the heating and cooling of the calorimeter core are studied. The differences of transient parameters in measurement and calibration operating regimes of the calorimeter are described. Initial data for design of the calibration model of the isoperibolic calorimeter are determined. It is recommended to take into account the nonlinear thermal and physical characteristics of the core materials.
\end{abstract}

\section{Statement of the problem}

Monitoring of energy parameters of radiation is the dominant type of measurement in the field of laser metrology. In the power range above several watt fractions, the minimum measurement uncertainty is provided by calorimetric primary measuring transducers (PMTs) calibrated by the method of electrical substitution. Reducing the uncertainty of calibration of measuring transducers is an important part of the task of improving the national measurement standards of Ukraine.

\section{Analysis of the state of the problem}

The fundamentally unrecoverable contribution to the uncertainty budget of calibration of calorimetric PMTs is made by the nonequivalence of substitution of laser radiation by calibration heat flux.

The nonequivalence of substitution is caused by the following sources:

- Asymmetry of the PMTs design for measurement and calibration processes.

- Diversity in space of the receiving area and the calibration heating coil of the measuring transducer.

- Inequality of irradiated and heated areas of the receiving element.

- Inequality in the volumes of the PMTs heating winding and the absorption region of the radiation of the receiving element.

- Difference between the heat transfer mechanisms from the irradiated and heated surfaces of the receiving element.

- Difference in heat exchange conditions for irradiated and heated surfaces of the receiving element.
- Difference in time characteristics of heat release during irradiation and heating of the receiving element.

- Dependence of the thermal and physical characteristics of the material of the receiving element from the temperature.

- Inequality of the thermal and physical characteristics of the materials of the receiving element and the heating winding.;

- Need for electrical insulation of the heating winding.

- Uncertainty of the location of the measuring point of the effective temperature of the receiving element.

For the present, there are no proven methods for registration of the nonequivalence of substitution in the literature.

The non-homogeneous internal structure of the calorimetric measuring transducer, the non-linearity of the processes occurring in it, and the multifactor calibration process make the development of reference PMTs much more difficult. In order to simplify the determination of the optimum parameters of the measuring transducer, its adequate model can be developed that will allow to predict the PMT reaction on a specified external influence, with the specified action being either measured laser radiation or change of environmental parameters.

The task of building a model does not have a singlevalued solution, since different theoretical approaches will lead to unequal models. The most valuable are the models reflecting, to a certain extent, the internal structure of PMT and its organization.

Such a model will allow to choose and justify the structural scheme of PMT, consisting of the simplest well-studied idealized elements.

*Corresponding author: vladimir.skliarov@metrology.kharkov.ua 


\section{Target of the work}

The target of this work is to obtain the initial information for building a model of a measuring transducer by experimental determining the parameters of the laser radiation power measurement process and the PMT calibration process.

\section{Description of the experiment}

The measurement process of the laser radiation power consists in applying the measured radiation to the PMT receiving element, waiting for the end of the transient process, and measuring the output signal of the thermocouple battery. The calibration process differs from the measurement process only in that the calibration thermal flux generated by the internal electric heater is applied to the receiving element instead of the measured optical radiation.

An experimental study of a calorimetric PMT was carried out at the installation shown in Fig. 1, which included ATC-C3000-500-AMF-ATS-03H diode laser $(\lambda=0.806 \mu \mathrm{m})$ with LDD-10 control device, DM 3559 and DT-830B multimeters, JPS-3030 calibration voltage source and a computer.

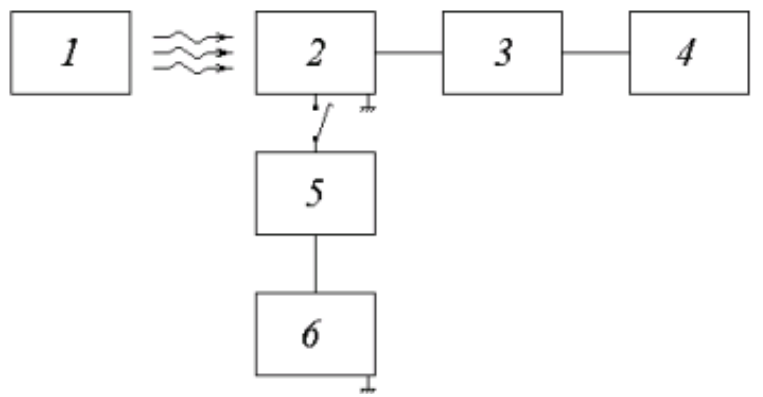

Fig. 1. The scheme of the experimental installation

1 - laser, 2 - PMT, 3 - DM 3559 millivolt meter, 4 - computer,

5 - DT-830B multimeters, 6 - voltage source.

The experimental procedure involved applying optical radiation of a constant power to the PMT receiving element and recording the voltage quantity $U_{i}$ of the PMT output electric signal from the moment the radiation was applied until the setting of the PMT stationary thermal regime. After reaching the steadystate thermal regime, the radiation source was turned off and the PMT output signal was registered until the moment of complete cooling of the receiving element. Then the power of the optical radiation was changed and the heating-cooling measurement cycle, and the recording of the PMT output signal were repeated.

The similar measurement cycle was performed when the calibrated heat flux was applied to the PMT receiving element.

An essential condition for recording the PMT output signal is the equidistance of the measurement moments in time. The measurement period was set by the internal generator of the multimeter and was $0.393 \mathrm{~s}$.

The typical graphs of the recorded transient processes are shown in Fig. 2.

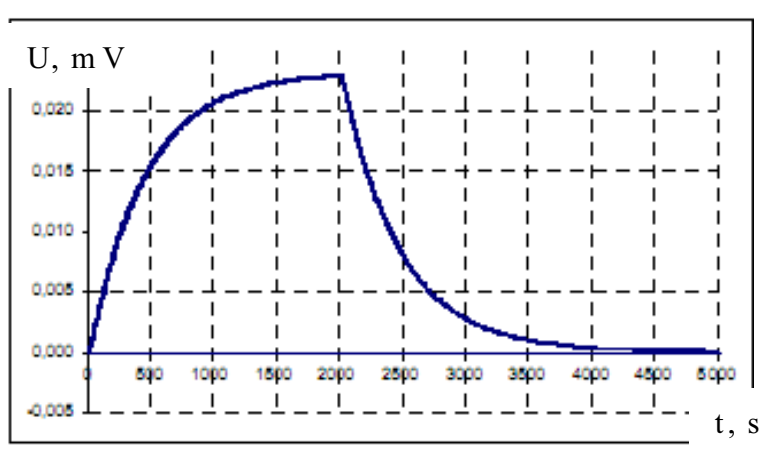

a) measured optical radiation

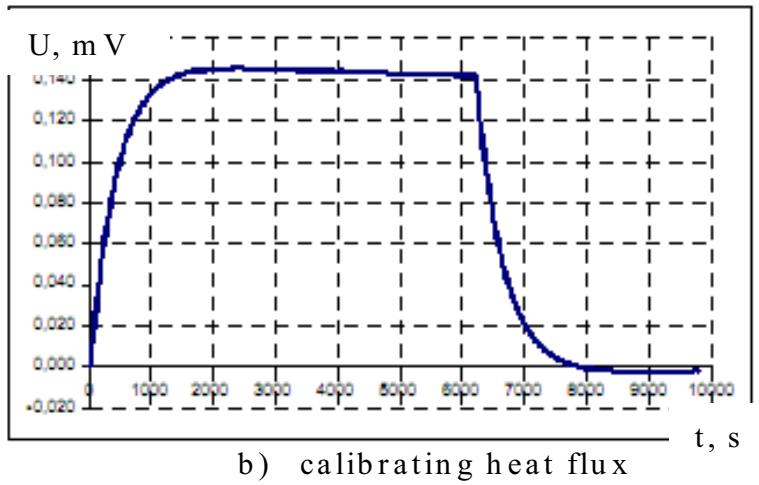

Fig. 2. The change in the output signal of the calorimetric transducer during the measurement cycle.

It is known from the theory [1] that transient processes in linear systems of the $n$-th order, which arise under external influence approximated by the Heaviside step function, are described by the sum of $n$ exponents. From this, it follows that any linear system can be represented in the form of a model containing some set of simplest structural elements of the first order.

In accordance with the above, processing of the accumulated data array [2] was conducted in order to obtain correct values of equivalent time constants for transient processes.

The well-known procedure for approximating the experimental graphs of the studied transient processes by an exponential polynomial [3] proved to be ineffective, since it did not allow us to filter out the failed and noisy results of measurements, and, most importantly, due to the proximity of the transient process to the exponential, the matrix of the coefficients of the normal equations was close to degenerate one distorted by malfunctions and noise, and, consequently, the roots of the normal equations were calculated with a large error, which leaded to an unacceptably large error in determining the time constants.

The direct calculation of the time constant of the transient process when heating (cooling) of the PMT receiving element was more effective by the following algorithm:

- By the array of quantities $U_{i}$ measured during the transient process, the array of quantities $\Delta U_{i}=U_{i+1}-U_{i}$ was calculated.

- By the array of quantities $\Delta U_{i}$, the array of quantities $\ln \Delta U_{i}$ was calculated.

- By the array of quantities $\ln \Delta U_{i}$, the array of quantities $\Delta F_{i}=\ln \Delta U_{i+1}-\ln \Delta U_{i}$ was calculated. 
- For the array of quantities $\Delta F_{i}$, the linear trend equation $\Delta F i=f(i)$ was calculated using the least squares method.

- The time constant of the transient process was calculated as the reciprocal to the constant term of the linear trend equation, and was reduced to the time units by multiplying by the sampling period of quantities $U_{i}$ in seconds.

For an ideal exponential transition process, the linear trend calculated by this algorithm is a straight line parallel to the abscissa axis and displaced down from it by a quantity reciprocal to the time constant of the exponent.

An example of the experimental graph $\Delta F i=f(i)$ for the calibrating transition process is shown in Fig. 3.

The linearity of the graph of the exponential region of the transient process indicates that, in the first approximation, the calorimetric PMT is a linear system of the first order. The coefficient of nonequivalence of substitution for the PMT sample studied in the experiment is equal to 1.023 with a confidence probability of 0.85 . Estimation of the coefficient of the substitution nonequivalence coincides with the value obtained with direct measurements of the energy characteristics of the laser radiation and its substitutional thermal influence of the electric current, taking into account the PMT blackness factor.

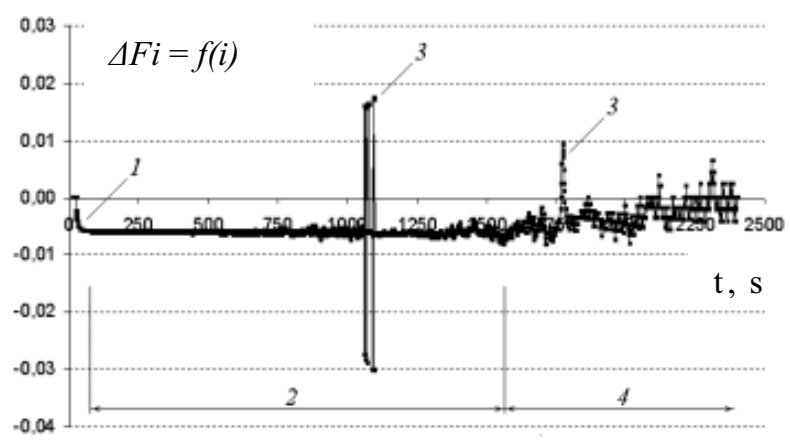

Fig. 3. Experimental dependence of the characteristics of the calibrating transition process from time

1 - initial non-exponential region of the transient process, 2 exponential region of the transient process, 3 - malfunctions of the measuring equipment, 4 - region of dominance of the measurement noises.

The values of the time constants of transient processes obtained in the experiment, averaged over the entire data array, are given in Table 1.

Table 1. The experimental values of the time constants of transient processes.

\begin{tabular}{|c|c|c|c|}
\hline \multicolumn{4}{|c|}{ The time constant of the transient process, $\mathrm{s}$} \\
\hline \multicolumn{2}{|c|}{ Measurement } & \multicolumn{2}{c|}{ Calibration } \\
\hline Heating & Cooling & Heating & Cooling \\
\hline 178.73 & 182.78 & 167.76 & 190.66 \\
\hline
\end{tabular}

\section{Conclusions and recommendations}

The obtained experimental results give grounds to state that the process of measuring the power of laser radiation can be described by an inhomogeneous differential equation of the first order. The calibration process for the isoperibolic calorimeter, due to a drop of the pulse, should be described by an inhomogeneous differential equation of the second order.

For further reduction of uncertainty of the PMT calibration, more complex model should be built based on the obtained initial data, in which the following experimental facts should be taken into account:

- The time constant of the transient heating process of the receiving element is bigger during measurement than during calibration.

- The time constant of the transient cooling process of the receiving element when measuring is smaller during measurement than during calibration.

- The time constants of all transient processes, measured in successive cycles, decrease monotonically.

The complication of the structure of the model, taking into account the nonlinearity of the thermal characteristics of the structural materials, requires joint application of the nonlinear heat equation and one of the numerical methods for the calculation of the temperature field of the receiving element of the calorimeter.

It should be noted that in order to simplify the model of the calibration process of the isoperibolic calorimeter, it is advisable to eliminate the drop of the calibration pulse by changing the design of the calorimeter.

The authors are grateful to Prof. Dr. Pavel Neyezhmakov for his valuable discussion and suggestions concerning the results of modeling for calibration of primary measuring converter by using of the initial data. The problems of adaptation and application of these results for international comparisons on the COOMET Projects were discussed.

\section{References}

1. M.F. Gardner, J.L. Burns. Transient processes in linear systems with lumped parameters. MoscowLeningrad, GITTL, 1949, 528 pp.

2. K.I. Muntyan, E.P. Timofeev. Investigation of the error of substitution of the optical signal by electrical one during calibration of calorimetric transducers. Lighting and power engineering, No. 2(22), 2010, pp. 4 - 11.

3. Ya.S. Bezikovych. Approximate calculations. M.: Gostekhizdat, 1949, 463 pp. 